\title{
Tone language experience enhances sensitivity to melodic contour
}

\author{
Evan D. Bradley \\ Department of Linguistics and Cognitive Science, University of Delaware
}

Pitch/F0 is used in language for lexical tone, and is a primary component of music. Musicians exhibit neurophysiological and behavioral enhancement of lexical tone perception (Alexander et al., 2005, Lee \& Hung, 2008; Wong \& Perrachione, 2007) due to more accurate tracking of pitch movement (Chandrasekaran et al., 2009). Likewise, tone language experience influences music perception (Stevens et al., 2004; Pfordresher \& Brown, 2009; Alexander et al., 2011), but these effects have not been fully linked to perceptual properties relevant to language and music. This study links acoustic dimensions of lexical tones with those of musical melodies, providing a possible explanation for these crossover effects which is consistent with theories of perceptual learning (Ahissar et al., 2009; Patel, 2008).

\section{Background}

The pitch content of lexical tones is perceived along at least three dimensions: height - average F0; direction of F0 change; and slope - rate of F0 change. Languages rely on these to various degrees depending on their tonal inventories, and speakers vary in sensitivity: non-tone speakers generally attend to height, whereas tone speakers rely more on pitch movement, but vary in relative sensitivity to the latter two dimensions (Gandour \& Harshman, 1978; Gandour, 1983; Khouw \& Ciocca, 2007). These perceptual differences have neural correlates (Krishnan et al., 2005; Chandrasekaran et al., 2007) and consequences for L2 learning (Francis et al., 2008; Wong \& Perrachione, 2007).

Melody perception can also be factored into several dimensions, which are analogous to those for tone: $\boldsymbol{k e} \boldsymbol{y}$ - the range the melody is played in, analogous to height; contour - the direction of change between notes (up/down/none), analogous to direction; and interval - the size of change between notes

(e.g., major third), analogous to slope. These are perceptually dissociable and altered by musical training (Massaro et al., 1980; Fujioka et al., 2004). These melodic properties are argued to share perceptual resources with the analogous phonetic properties of tone. Recognition of this correspondance provides a framework for further specifying and explaining mutual influence between lexical tone and melody.

\section{Hypotheses}

Previously observed crossover effects between language and music are consistent with the Shared Sound Category Learning Mechanism Hypothesis (Patel, 2008), which suggests that music and language share auditory processing resources and mechanisms for learning sound categories, but that the representations of units and rules for each system are separate. This formulation is compatible with Reverse Hierarchy Theory (RHT) (Ahissar et al., 2009), a model of learning in which learning begins at the highest neural level which can solve a perceptual task, with feedback tuning sensory inputs in ways relevant to the task; these tuned resources are then available to other tasks drawing on the same perceptual properties.

If pitch perception in language and music share perceptual resources, then tone language speakers' enhanced sensitivity to direction and slope will drive more accurate perception of the contour and intervals of melodies in corresponding ways; specifically: (1) speakers of languages which rely heavily on direction to perceive tone will more accurately perceive melodic contour than those who don't; (2) speakers of 
languages which rely heavily on slope to perceive tone will more accurately perceive melodic interval than those who don't.

These hypotheses were tested by comparing speakers of a nontone language (English) with speakers of a tone language (Mandarin). Direction and slope are important cues to Mandarin tone identification (Gandour, 1983; Shen \& Lin, 1991), while height is of relatively less importance (Gandour, 1983; Francis et al., 2008). Thus, differences are expected to occur between Mandarin and English speakers on only some aspects of melodic structure: (1) Mandarin speakers will more accurately perceive melodic contour than English speakers; (2) Mandarin speakers will more accurately perceive melodic interval than English speakers; (3) Mandarin and English speakers will not differ in perception of melodic key.

\section{Method}

\section{Participants}

Participants were native speakers of Mandarin $(n=17)$ or English $(n=20)$. No participant had experience with another tone language, and none were musicians, defined as less than five years experience with a musical instrument or voice.

\section{Procedure}

Participants took a modified subtest of the Musical Ear Test (MET) (Wallentin et al., 2010). Participants heard pairs of piano melodies responded if they detected changes to melodies which were keychanging, contour-changing, or contour-preserving (interval-changing).

\section{Analysis}

The MET was modeled as a signal detection task, in which the signal which must be detected is the difference between the melodies. A measure of discriminability was computed for each violation type per subject $\left(A^{\prime}\right.$, shown in (1), where hit refers to accuracy on "different" trials, and $f a$ to false alarm rate, or incorrect responses to "same" trials). $A^{\prime}=1$ indicates perfect discrimination; $A^{\prime}=0.5$ indicates chance performance. $A^{\prime}$ scores were entered into a mixed-effects analysis of variance with Violation Type (interval, contour, key) as a within-subjects factor and Subject L1 as a between subjects factor.

$$
A^{\prime}=1 / 2+\frac{(h i t-f a) *(1+h i t-f a)}{4 * h i t *(1-f a)}
$$

\section{Results}

There was a significant main effect of Violation Type $(F(2,70)=111.39, p<.001)$, with interval being the most difficult violation to detect for both Mandarin and English listeners. Mandarin and English listeners differed significantly in overall MET performance $(F(1,35)=7.96, p<.01)$, but this was not uniform over the violation types; there was a significant interaction between Subject L1 and Violation Type $(F(2 / 70)=3.21, p<.05)$, with Mandarin subjects showing greater discrimination of contour $\left(p_{a d j}<.05\right)$ and interval $\left(p_{a d j}<.005\right)$ changes compared to English speakers. ${ }^{1}$ Mandarin and English speakers did not differ in discrimination of key changes. Results are summarized in Table 1 and Figure 1 .

\footnotetext{
${ }^{1}$ LSA meeting slides reported a significant effect on contour, and a trend approaching significance on interval; this reflected probabilities corrected for irrelevant comparisons not made, and thus was overly conservative.
} 


\begin{tabular}{ccccc}
\hline & interval & contour & key & total \\
\hline English & $0.59(.15)$ & $0.85(.07)$ & $0.90(.06)$ & $0.78(.17)$ \\
Mandarin & $0.70(.14)$ & $0.92(.06)$ & $0.91(.06)$ & $0.84(.14)$ \\
\hline
\end{tabular}

Table 1: Mean discrimination $\left(A^{\prime}\right)$ of melody violation types by Mandarin and English subjects (SD in parentheses).

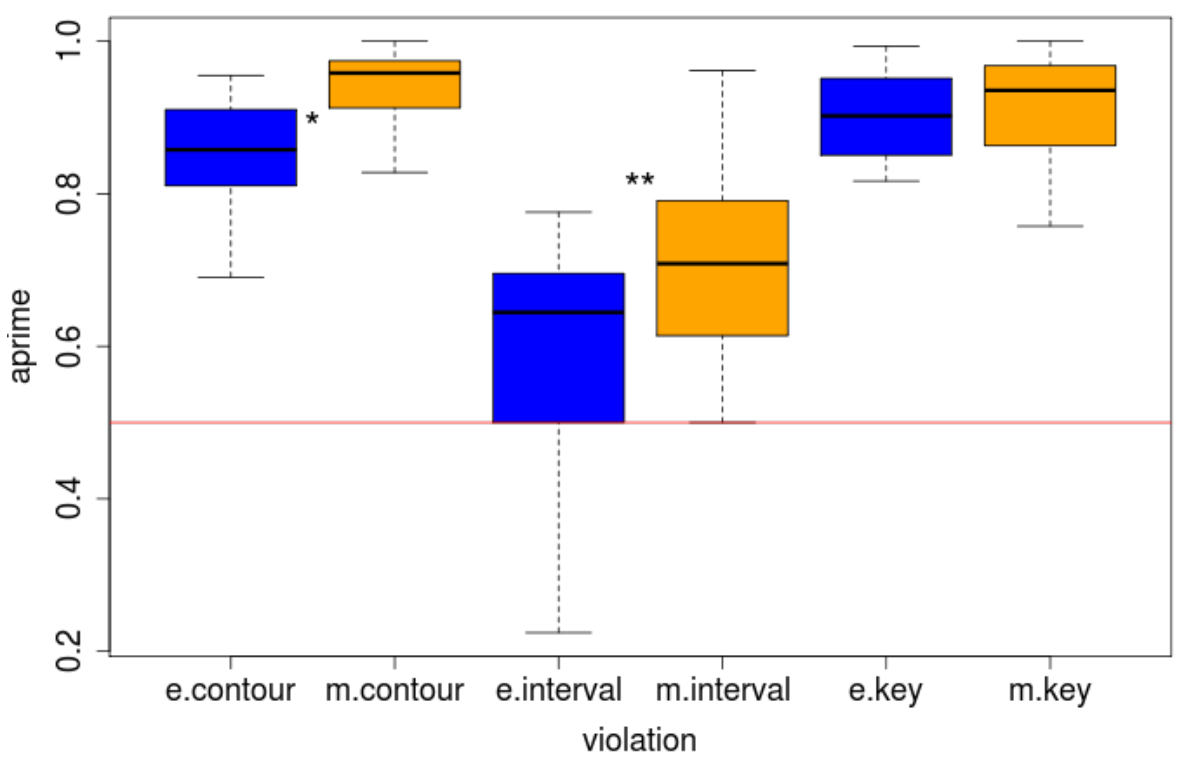

Figure 1. Discrimination of melody violation types by Mandarin (orange) and English (blue) subjects. Red line indicates chance discrimination $\left(A^{\prime}=.5\right)$; '*' $p<.05$; '**' $p<.005$.

\section{Summary}

Although Mandarin subjects performed better on the MET, tone language experience does not cause general improvement of melody perception. Mandarin speakers showed advantages only in discrimination of contour and interval, which correspond to direction and slope, properties relevant to discriminating the tonal inventory of Mandarin. Mandarin and English speakers did not differ in discrimination of key. These results are consistent with RHT (Ahissar et al., 2009), in which domain-general neural resources are tuned according to task demands. Mandarin speakers experience sensory tuning only to those phonetic properties required by their tonal inventory, and thus effects on their melody perception are limited to corresponding properties reling on the same resources

These findings further previous studies demonstrating effects of tone language experience on melody perception by more explicitly linking individual dimensions of pitch perception between melody and lexical tones, and suggests that, at least at this level, language and music share perceptual and neural resources. Future work on additional languages will strengthen the proposed correspondance between structural properties of language and music.

\section{References}

Ahissar, M., Nahum, M., Nelken, I., \& Hochstein, S. (2009). Reverse hierarchies and sensory learning. Philosophical Transactions of the Royal Society of London. Series B, Biological Sciences, 364(1515), 285-99. http://rstb.royalsocietypublishing.org/content/364/1515/285 
Alexander, J. A., Bradlow, A. R., Ashley, R. D., \& Wong, P. C. (2011). Music-melody perception in tone-language and non-tone-language speakers. In Psycholinguistic representation of tone. Hong Kong. http://www.sfu.ca/ jaa37/index_files/Alexander_et_al_2011.pdf

Alexander, J. A., Wong, P. C., \& Bradlow, A. R. (2005). Lexical tone perception in musicians and non-musicians. In Proceedings of the 9th European conference on speech communication and technology. Lisbon. http://www.isca-speech.org/archive/interspeech_2005/i05_0397.html

Chandrasekaran, B., Gandour, J. T., \& Krishnan, A. (2007). Neuroplasticity in the processing of pitch dimensions: a multidimensional scaling analysis of the mismatch negativity. Restorative Neurology and Neuroscience, 25(3-4), 195-210. http://www.ncbi.nlm.nih.gov/pubmed/17942999

Chandrasekaran, B., Krishnan, A., \& Gandour, J. T. (2009). Relative influence of musical and linguistic experience on early cortical processing of pitch contours. Brain and Language, 108(1), 1-9. http:// WWW.ncbi.nlm.nih.gov/pubmed/18343493

Francis, A. L., Ciocca, V., Ma, L., \& Fenn, K. (2008). Perceptual learning of Cantonese lexical tones by tone and non-tone language speakers. Journal of Phonetics, 36(2), 268-294. http://linkinghub .elsevier.com/retrieve/pii/S0095447007000289

Fujioka, T., Trainor, L. J., Ross, B., Kakigi, R., \& Pantev, C. (2004). Musical training enhances automatic encoding of melodic contour and interval structure. Journal of Cognitive Neuroscience, 16 (6), 1010-1021. http://www.ncbi.nlm.nih.gov/pubmed/15298788

Gandour, J. T. (1983). Tone perception in Far Eastern languages. Journal of Phonetics, 11(2), $149-175$.

Gandour, J. T., \& Harshman, R. A. (1978). Crosslanguage differences in tone perception: a multidimensional scaling investigation. Language and Speech, 21(1), 1-33.

Khouw, E., \& Ciocca, V. (2007). Perceptual correlates of Cantonese tones. Journal of Phonetics, 35(1), 104-117. http://linkinghub.elsevier.com/retrieve/pii/S0095447005000586

Krishnan, A., Xu, Y., Gandour, J. T., \& Cariani, P. (2005). Encoding of pitch in the human brainstem is sensitive to language experience. Cognitive Brain Research, 25(1), 161-168. http://www.ncbi.nlm .nih.gov/pubmed/15935624

Lee, C.-Y., \& Hung, T.-H. (2008). Identification of Mandarin tones by English-speaking musicians and nonmusicians. The Journal of the Acoustical Society of America, 124(5), 3235-3248. http://www.ncbi .nlm.nih.gov/pubmed/19045807

Massaro, D. W., Kallman, H. J., \& Kelly, J. L. (1980). The role of tone height, melodic contour, and tone chroma in melody recognition. Journal of Experimental Psychology: Human Learning and Memory, 6(1), 77-90. http://www.sciencedirect.com/science/article/pii/S0096151507604174

Patel, A. D. (2008). Language, music, and the brain. Oxford: Oxford University Press.

Pfordresher, P., \& Brown, S. (2009). Enhanced production and perception of musical pitch in tone language speakers. Attention, Perception, \& Psychophysics, 71, 1385-1398. http://dx.doi.org/10.3758/APP .71 .6 .1385

Shen, X. S., \& Lin, M. (1991). A perceptual study of Mandarin tones 2 and 3. Language and Speech, 34(2), 145-156. http://las.sagepub.com/cgi/content/abstract/34/2/145

Stevens, C., Keller, P., \& Tyler, M. (2004). Language tonality and its effects on the perception of contour in short spoken and musical items. In S. D. Lipscomb, R. Ashley, R. O. Gjerdingen, \& P. Webster (Eds.), Proceedings of the 8th international conference on music perception 8 cognition. Adelaide, Australia: Causal Productions. http://en.scientificcommons.org/48737698

Wallentin, M., Nielsen, A. H., Friis-Olivarius, M., Vuust, C., \& Vuust, P. (2010). The Musical Ear Test, a new reliable test for measuring musical competence. Learning and Individual Differences, 20, 188-196. http://www.sciencedirect.com/science/article/pii/S1041608010000233

Wong, P. C., \& Perrachione, T. K. (2007). Learning pitch patterns in lexical identification by native English-speaking adults. Applied Psycholinguistics, 28(4), 565-585. http://journals.cambridge.org/ abstract_S0142716407070312 